\title{
Нейросети, глубокое обучение, машинное зрение в сельском хозяйстве. Краткий обзор для 2021 года
}

Рахматулин Ильдар, к.т.н

Южно-Уральский государственный университет, кафедра сетей и систем электрических станций, г. Челябинск, Россия, 454080

\section{ildar.o2010@yandex.ru}

\section{Аннотация}

Беспрецедентный прогресс в области глубокого обучения повлиял на многие отрасли, в том числе и на сектор сельского хозяйства. Использование нейронных сетей в агропромышленной деятельности в задаче распознавания различных культур и сорняков - новое направление с историей менее 10 лет. Десятки новых нейронных сети появляются каждый год, но отсутствие каких-либо стандартов существенно затрудняет понимание реальной ситуации использования нейронной сети в аграрном секторе. В рукописи мы проанализировали исследования за последние 10 лет по использованию нейронных сетей для классификации и отслеживание посевов и сорняков в сельском хозяйстве. Мы представили анализ результатов использования различных нейронной сети для задач классификации и отслеживания. Наконец, мы сделали рекомендации по использованию нейронных сетей в задачах распознавания культивируемого объекта и сорняков.

Ключевые слова: машинное зрение в сельском хозяйстве, машинное обучение в сельском хозяйстве, распознавание сорняков, глубокое обучение для сельского хозяйства, умная ферма

\section{1. Введение}

В последние годы использование искусственного интеллекта быстро расширилось. Исследователи из разных областей науки используют на практике функционал нейронных сетей машинного обучения и машинного зрения. Но нейронные сети обладают очень продвинутой функциональностью и разнообразием, и если исследователь не принимал непосредственное участие в работе с нейросетями на протяжении последних лет, тогда ему это очень сложно эффективно подобрать правильную модель нейронной сети. Поэтому задача этой рукописи предоставить информацию о текущих тенденциях в области нейронных сетей в контексте сельского хозяйства.

Мы рассматривали только завершенные исследования, в которых представлены результаты тестирования нейронных сетей. По своим характеристикам эти результаты должны были подходить для полевого применение. Поиск статей по ключевым словам проводился в следующих издательствах: Elsiver, Тейлор и Фрэнсис, Springer, Wiley, Informa. Поиск по ключевым словам также выполнялся в поиске Google. engine и scholar.google.com за последние 10 лет. 
Следующие статьи аналогичны по содержанию этой рукописи. Ip et al. [1] предоставил обзор значительных исследований в области защиты растений с использованием больших данных с упором на борьбу с сорняками. Авторы рассмотрели методы машинного обучения для анализа больших данных. Ван и др. [2] представили аналогичную статью, в которой резюмировали достижения в обнаружении сорняков наземными методами машинного зрения и изображения обработка. Эти рукописи описывают исследования без какого-либо систематического подхода, согласно различным критериев, из-за которых довольно сложно представить полную картину использования нейронных сети в аграрном секторе.

\section{1. Распознавание сорняков без нейронных сетей}

Задача распознавания сельскохозяйственных культур и сорняков уже стояла перед фермерами несколько десятилетий назад. В результате чего есть много видов исследований, которые в контексте нейронных сетей теперь утратили свои прежние актуальность, но в то же время, за счет новаторских технических идей и нестандартных математических решения, они несут определенную научную ценность. Nejati et al. [3], для определения сорняков в моркови использовали метод активной стереоскопии на основе кодированного структурированного света с временным мультиплексированием. Это довольно дорогой метод, и его можно использовать только для того чтобы сравнить результаты с данными, полученными с помощью нейронных сетей. Nejati et al. [4] использовали процесс идентификации сорняков для быстрого преобразования Фурье плотности края листа. Этот способ может быть реализована как вспомогательная функция в процессе подготовки изображений для нейронных сетей. В какой-то степени могут быть полезны работы, которые напрямую не относятся к теме машинного зрения, но представляет интерес, благодаря наличию физических исполнительных механизмов, которые могут использоваться в составе устройств с машиным зрением. Rueda-Ayala et. др. [5], Утстумо и др. [6], Альба и др. [7], Меландер и др. [8] и Сыров [9] представили типичные устройства для механической борьбы с сорняками, которые можно легко соединить с различными системами на основе нейронных сетей.

\section{2. Предварительная обработка изображений в задачах распознавания сорняков}

Качество работы нейронной сети напрямую зависит от предварительной обработки изображений. Следовательно, этот аспект - один из самых важных в области машинного зрения. Нет прямых критериев с указанием того, как обрабатывать изображения, поскольку каждый случай уникален и требует отдельного подхода. Panqueba et al. [10], использовали функцию сегментации изображения и использование нейронной сети, обученной с сигмовидным типом функции активации. Они реализовали скрипт, который автоматически обрабатывает изображения перед использованием нейронной сети. Редди и др. [11] для аналогичных задач удалось добиться положительного результата только с такими фильтрами, как Гауссовский и Лапласианский. Невозможно сравнить эти методы на наборах данных автора, так как они не представлены в 
открытом доступе. Вихрам и др. [12] и Паша и др. al [13] использовал функцию cv2.inRange () библиотеки OpenCV в отслеживание задач. Это очень простой и популярный метод, который с высокой точностью работает в лабораторных условиях. То есть в условиях, когда можно специально создать необходимый цветовой контраст. Многие работы, в которых авторы не используют популярные библиотеки компьютерного зрения для реализации процесс обработки изображений. Эти работы предполагают реализацию обработки изображений за счет математических формул на основе Matlab или другого программного обеспечения. Например, Гарсия-Сантильян и др. [14], реализовал процесс сегментации изображения на основе использования индекса растительности и линейного наименьшего регрессия квадратов для исправления линии. Мурават [16] использовал двоичную классификацию для подготовки изображений перед использованием нейронной сети. Michael et al. [18] разработали программное обеспечение для обработки изображений. Реализуемый алгоритм, основан на эрозии с последующим алгоритмом сегментации расширения. Но в этих исследованиях, проверка другими методами не проводилась. Чтобы повторить эксперимент, требуется установка программного обеспечения. Более того, эти методы крайне сложно реализовать. совместно с нейронными сетями. Необходимо использовать стационарный компьютер с достаточной мощностью GPU для выполнения вычислений. В отличие от этих работ, Murawwat et al. [15] использует процедуры машинного обучения для реализации опорных векторных машин (SVM) и BLOB объекты для задачи обнаружения урожая с низким энергопотреблением ЦП. Bhongal et al. [17] использовали аналогичный стандарт функции и использовали сегментацию и последующее использование нейронной сети для обработки изображений, так же сделали и Амбика и др. [19] и Феррейра и др. [20]. Общим недостатком описанных выше работ является отсутствие полного обзора литературы, поэтому существует много дублирующих работ. В таких работах аналогично описывается процесс предварительной обработки изображений или используется аналогичная модель нейронной сети с такой же функцией активации нейрона. Стоит отметить, что география исследований обширна, как по странам исследователей, так и по культурам и сорнякам. Для проверки модели в работах искусственно созданные макеты с ярко выраженным цветовым контрастом, что не позволяет оценить, как эти модели работают на практике. Общей чертой этих работ и, в меньшей степени, последующих работ является излишне подробное описание предварительной обработки изображений, которая, хотя и является важным компонентом исследований, связанных с машинным зрением, но все же не несет в себе научной новизны. В то же время оценка правильности предварительной обработки в описываемых работах - сложная задача, поскольку каждый случай уникален и зависит от поставленных задач и количества отправленных на обработку фотографий. По результатам анализа можно сделать вывод, что по большей части используется не весь возможный функционал для предварительной обработки изображений, например, который можно использовать в библиотеке OpenCV. Ниже приведен список, который полезен для предварительной обработки изображений. В скобках указаны операторы, используемые для вызова объекта на языке программирования Python в библиотеке OpenCV. 


\section{Обработка изображений.}

\section{По цвету:}

- color filters (cv2.createTrackbar, cv2.cghhnbnmlootroiesxxxvtColor, color_image[:,:,n])

- color detection (cv2.inRange, cv2.findContours);

\section{- Smoothing Images:}

- Convolution (cv2.filter2D);

- Image Blurring (cv2.blur);

- Gaussian Filtering (cv2.GaussianBlur);

- Median Filtering (cv2.medianBlur);

- Bilateral Filtering (cv2.bilateralFilter);

- Laplacian (cv2.Laplacian).

- Morphological Transformations:

- Erosion (cv2.erode);

- Dilation (cv2.dilate);

- Opening (cv2.MORPH_OPEN);

- Closing (cv2.MORPH_CLOSE);

- Morphological Gradient (cv2.MORPH_GRADIENT);

- Top Hat (cv2.MORPH_OPEN);

- Black Hat (cv2.MORPH_BLACKHAT).

Edge Detection (cv2.Canny)

\section{Contours:}

- Contours （cv.drawContours）;

- Shapes (cv2.rectangle, cv2.minEnclosingCircle, cv2.circle, cv2.line);

- Fill (cv2.floodFill).

\section{Thresholding:}

- Binary (cv2.THRESH_BINARY);

- Binary_inv (cv2.THRESH_BINARY_INV);

- Trunc (cv2.THRESH_TRUNC);

- Tozero (cv2.THRESH_TOZERO);

- Tozero_inv (cv2.THRESH_TOZERO_INV).

\section{Geometrical image transformation:}

- Resize (cv2.resize);

- Translation cv2.warpAffine();

- Rotation (cv2.getRotationMatrix2D);

- Affine Transformation (cv2.getAffineTransform);

- Perspective Transformation (cv2.getPerspectiveTransform, cv2.warpPerspectiv).

\section{Image Derivatives:}


- Sobel derivatives (cv2.Sobel);

- Scharr derivatives (cv2.Scharr).

Использование этих функций позволит оценить и понять, какие элементы на изображении могут быть полезны для распознавания сорняков. Например, на рисунке 1 мы реализовали наиболее популярные функции для процесс распознавания изображений (https://github.com/Ildaron/OpenCVimage-preprocessing-python). Отделение растения на передней кромке от фона представляет определенные трудности. Решение состоит в том, чтобы выбрать правильный метод и правильные коэффициенты пороговой функции.

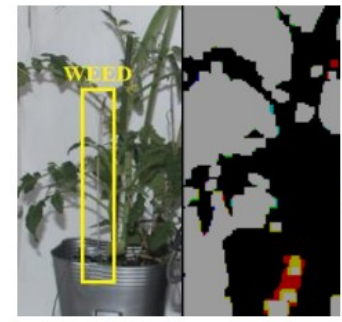

a

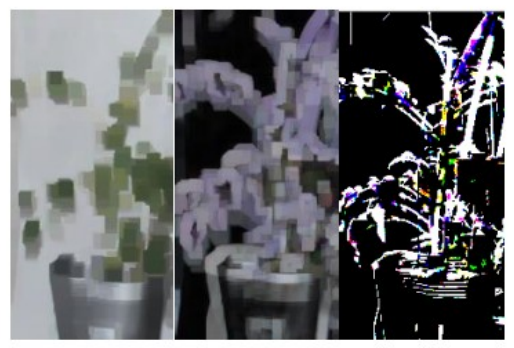

h

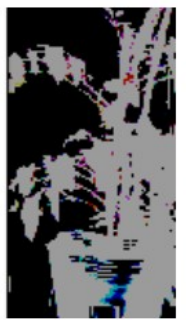

C

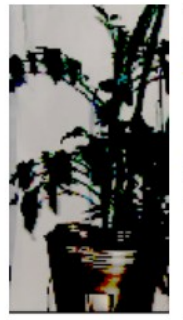

d

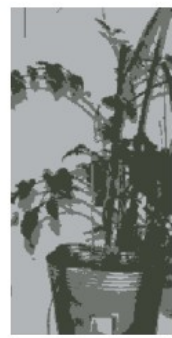

1

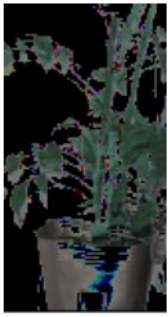

e

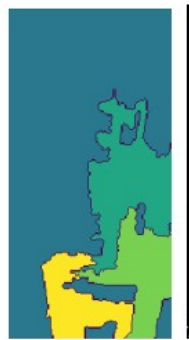

$\mathrm{m}$

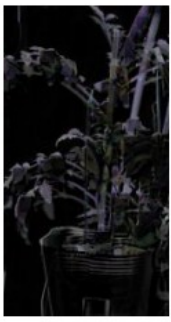

$\mathrm{f}$

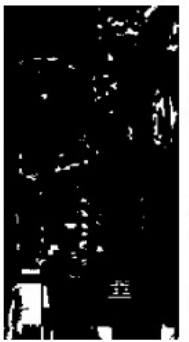

$\mathrm{n}$

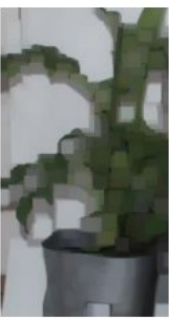

g

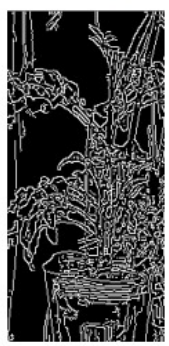

p

Рис.1. Распознавания изображений: a - Исходное изображение - сорняк и томат, b - image thresholding by cv2.threshold(img,127,155,cv2.THRESH_BINARY), c - image thresholding by cv2.threshold(img,127,155,cv2.THRESH_BINARY_INV), d - image thresholding by cv2.threshold(img,127,155,cv2.THRESH_TOZERO), e - image thresholding by cv2.threshold(img,127,155,cv2.THRESH_TOZERO_INV), f - morphological transformations by cv2.morphologyEx(img, cv2.MORPH_BLACKHAT, kernel1), g - morphological transformations by

cv2.morphologyEx(img, cv2.MORPH_OPEN, kernel2), h - morphological transformations by cv2.morphologyEx(img, cv2.MORPH_CLOSE, kernel2), j - morphological transformations by cv2.morphologyEx(img, cv2.MORPH_GRADIENT, kernel2), k -flood fill by cv2.floodFill(im_floodfill, mask, (0, 0), 255), l - segmentation by cv2.kmeans(pixel_values, k, None, criteria, 10, cv2.KMEANS_RANDOM_CENTERS), $\mathrm{m}$ - distance transform by cv2.distanceTransform(opening,cv2.DIST_L2,5), n - color detection by cv2.inRange(hsv, h_min, h_max)

$$
- \text { from }
$$

\section{3. Глубокое обучение в задачах распознавания сорняков}

В последние годы глубокое обучение в связи с доступностью вычислительной мощности стало широко использоваться в задачах машинного зрения. Сегодня использование глубокого машинного обучения является приоритетом в проблемах классификации и отслеживания, что 
подтверждается результатами соревнований на Kaggle (www.kaggle.com) и Image.net. Самая популярная нейронная сеть, используемая в задачах классификации, - это сверточная нейронная сеть (CNN). В рукописях авторы используют различные модели нейронных сети для классификации сорняков и задач по выращиванию культур. Как правильно, чтобы подтвердить эффективность

По предложенным методикам авторы приводят сравнения предложенной модели с другими моделями нейронные сети. Но каждый автор решает, насколько успешен предложенный метод в рукописи сам без всяких стандартов. Например, в следующих статьях использовались разные модели нейронных сетей. для задач распознавания сорняков. Bah et al. [32] рассмотрели как сеть CNN, так и остаточную сеть CNN для классификации изображений. Priya et al. [33] создали глубокую нейронную сеть с использованием библиотеки Keras. Potena et al. [34] использовали нейронную сеть CNN для пиксельной сегментации двоичного изображения, для извлечение пикселей и построение проекции трехмерных точек. Ахмед и др. [35], создали сеть на VGG16 и подробно обсудили принципы глубокого обучения и представили результаты экспериментов и испытаний. Sharma et al. [36] рассматривается остаточная сеть 101. Сеть и параметры которой настраиваются с помощью метода с прогрессивным изменением размера, со скоростью циклического обучения и функция потери фокуса. Zhang et al. [37] провели подробное сравнение сетей VGG и CCD для поиска сорняков.

Крайне редко в исследованиях встречается один и тот же тип сорняков, что делает невозможным непосредственное сравнить несколько рукописей. В описанных выше работах недостаточно аргументировано выбор моделей и критерии успеха очень условны. Можно сделать вывод, что с практической точки зрения эти исследования актуальны, но не приносят сколько-нибудь значимого научного достижения.

\section{4. Беспилотный летательный аппарат для задач распознавания сорняков}

Наиболее перспективным направлением использования нейронных сетей в задачах распознавания сорняков является возможность использования небольшой компьютерной системы с функцией машинного зрения в составе беспилотного летательного аппарата. В нескольких исследованиях описан процесс реализации использования беспилотного летательного аппарата для борьбы с сорняками. Так как в этом случае качество фото/видео ограничено, из-за нестабильного положение дрона то особенно важна предварительная обработка изображений. Хуанг и др. [38] использовали полною свёрточную сеть (FCN) для распознавания сорняков по изображениям, собранным устройством. Hameed et al. [39], использовали самолет для сбора информации об урожае. Многие виды исследований описывают метод с общим анализом продуктивности без точного распознавания урожая [40, 41].

На сегодняшний день не существует беспилотных летательных аппаратов, которые могли бы преодолеть трудности с распознаванием. культуры в масштабе сельскохозяйственной фермы и до сих пор обычно используются для представления общей картины урожай на поле. 
Перспективным направлением исследований в этой отрасли является использование одноплатного компьютера. Но использование методов глубокого обучения на самом популярном одноплатном компьютере - Raspberry PI из-за ограниченная оперативная память (1-4 ГБ) и из-за низкой скорости процессора 1,5 ГБ практически невозможна (ResNet>100 МБ, VGGNet> 550 МБ, AlexNet> 200 МБ, GoogLeNet> 30 МБ). Обнаружение в реальном времени с R-CNN, Fast R-CNN, Faster R-CNN, Yolo, RetinaNet имеют такую же проблему скорости распознавания. Решением может быть использование - NVIDIA Jetson TX1 и TX2 - специальная платформа для вычислений нейронных сетей. Главный недостаток - высокая стоимость. Компания STMicroelectronics сделала возможным использование методов глубокого обучения на микроконтроллерах. Они выпустили X-CUBE-AI - AI - пакет расширения для STM32CubeMX. Это расширение может работать с различными библиотеками глубокого обучения, такими как среды - Caffe, Keras, TensorFlow, Caffe, ConvNetJs и т. д. Благодаря этому нейронная сеть можно обучить на настольном компьютере с возможностью вычислений на графическом процессоре. После интеграции использовать оптимизированную библиотеку для 32-битного микроконтроллера STM32. В то же время, используя микроконтроллер вместо одноплатного компьютера можно значительно снизить энергопотребление. Поэтому использование нейронных сетей в составе беспилотного летательного аппарата имеет хорошие перспективы

\section{5. Компании-разработчики в задачах распознавания сорняков}

Несколько компаний предоставляют услуги по продаже автономных роботов для точечной прополки сельскохозяйственных культур. Компания Naio-technologies представила робота OZ WEEDING ROBOT. Bilberry Company - это разработка систем слежения, которые могут быть установлены на сельскохозяйственную технику для последующего пропуска сорняков. PrecisionHawk's разрабатывает автономные летательные аппараты с системами идентификации сорняков. Компания Blue River Technology размещает на веб-сайте информацию о разработках в области использования нейронных сетей в сельском хозяйстве. Ecorobotix представили WEEDING ROBОT. Карре разработал Робот АНАТИС. Все описанные выше компании по разработанным технологиям не предоставили документации и экспериментальных данных, по которым можно судить о результатах работы устройств. В лучшем случае, компании ограничились фрагментами видео-демонстрации устройств.

Стоит рассмотреть агро-маркет - это становится очень популярным местом для стартапов. Это подтверждается информацией о крупных финансовых вложениях в различные компании, занимающиеся разработкой информационных технологий для сельскохозяйственных предприятий. Работающих прототипов найти не удалось. Результаты исследований стартапов в силу объективных причин являются даже более закрытые, чем компании, описанные ранее, поэтому они не рассматривается в данном исследовании.

\section{6. Другие исследования в задачах распознавания сорняков}


Очень популярны рукописи, в которых представлен прототип роботизированного комплекса для автоматического удаления сорняков. в полях. Например, Sabanci et al. [21], Young et al. [22], Frasconi et al. [23], Kulkarni et al. [24], Бахшипур и др. [25], Kargar et al. [26], Potena et al. [27] и Слотер и др. [28] представили различные нейронные сети для диагностики сорняков и механизмы для их удаления. Недостаток этих работах - слишком много информации: механика, электроника, нейронные сети, робототехника. В результате кратко описывается реализация нейронных сетей. В отличие от этих статей в диссертации по данной тематике максимально подробно описаны все аспекты исследования. В диссертации [29] описан процесс разработки интеллектуального сельскохозяйственного автомобиля. Автор использовал машинное зрение , как устройство бифокальной визуализации, работающее в 2-х режимах - одновременная широкоугольная съемка изображение и примерная съемка телеобъективом. Для анализа наличия сорняков из телеобъективом были получены фотографии высокого разрешения, а широкоугольная камера позволила получить увеличение зоны наблюдения. Результат с высокой точностью получен с точки зрения поиска сорняков в поле с камеры, установленной на автомобиле. Но, из-за высокой стоимости камеры системы этот формат больше подходит для исследований, чем для последующей работы на поле. Дирманн [30] подробно показал преобразование изображений между нейронными слоями, рис.2. В работе используются стандартные методы предварительной обработки изображений и сверточная нейронная сеть.

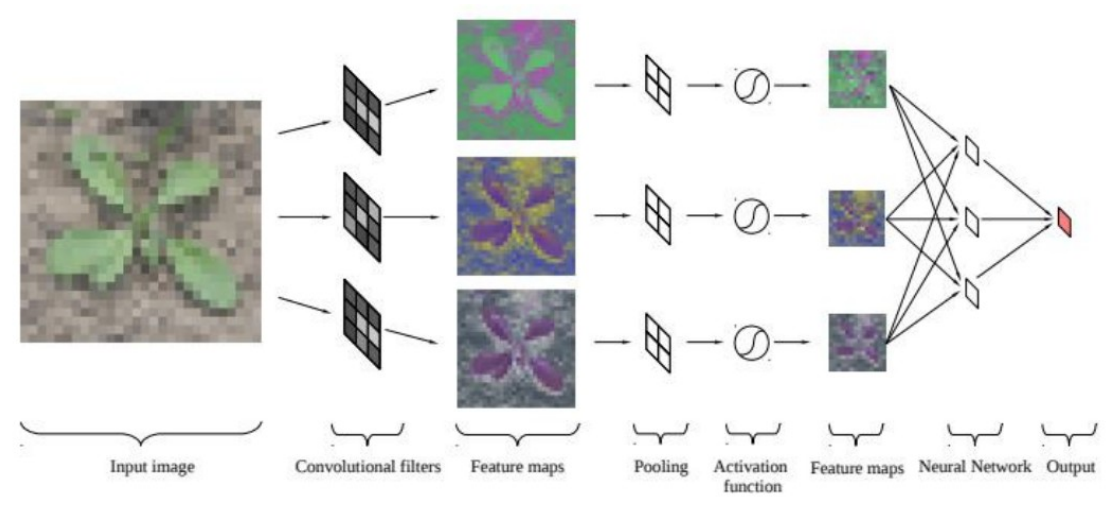

Рис. 2. Демонстрация модели CNN.

В отличие от этого тезиса Альба [31] использовал не самый популярный алгоритм, основанный на FuzzyC-means - метод классификационных задач. В диссертации исследована техника компьютерного зрения в реальном времени для надежного отслеживания всходов растений в полевых условиях. Были предложены различные методы сегментации.

\section{3. Обсуждение и выводы}

Обзор показал большое разнообразие типов нейронных сетей, используемых в процессе распознавания сорняков, где лидирующие позиции заняли разные реализации сверточной нейронной сети. 
Ниже приводится рекомендуемый список глубоких нейронных сетей для классификации изображений. Эти модели рекомендуется использовать в задачах классификации сорняков, рис. 3.

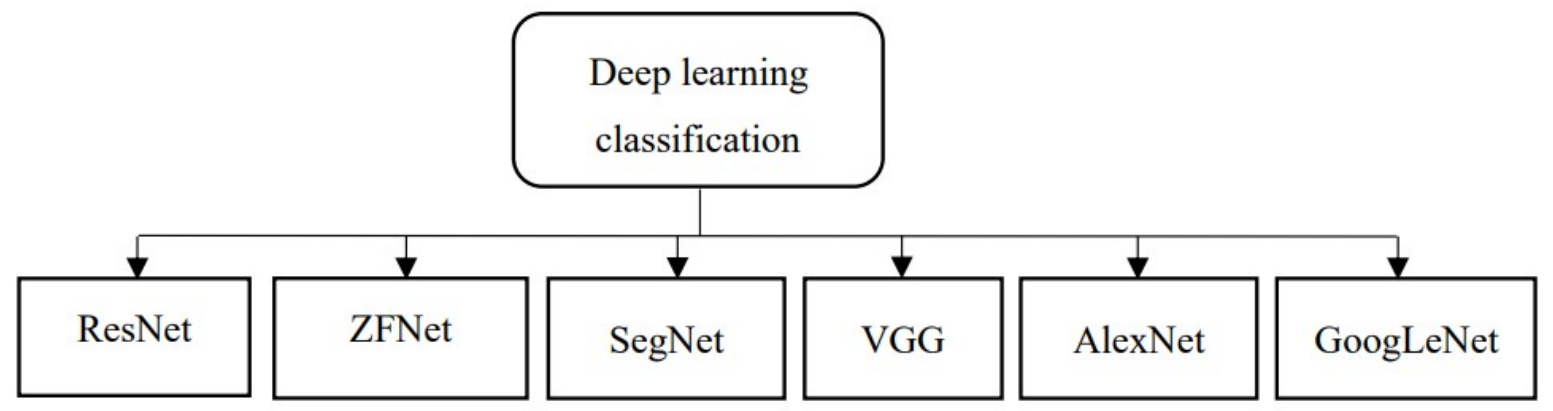

Рис. 3. Нейронные сети, рекомендуемые для классификации изображений при распознавании растений.

Для распознавания сорняков в видеопотоке рекомендуются следующие модели, рис. 4.

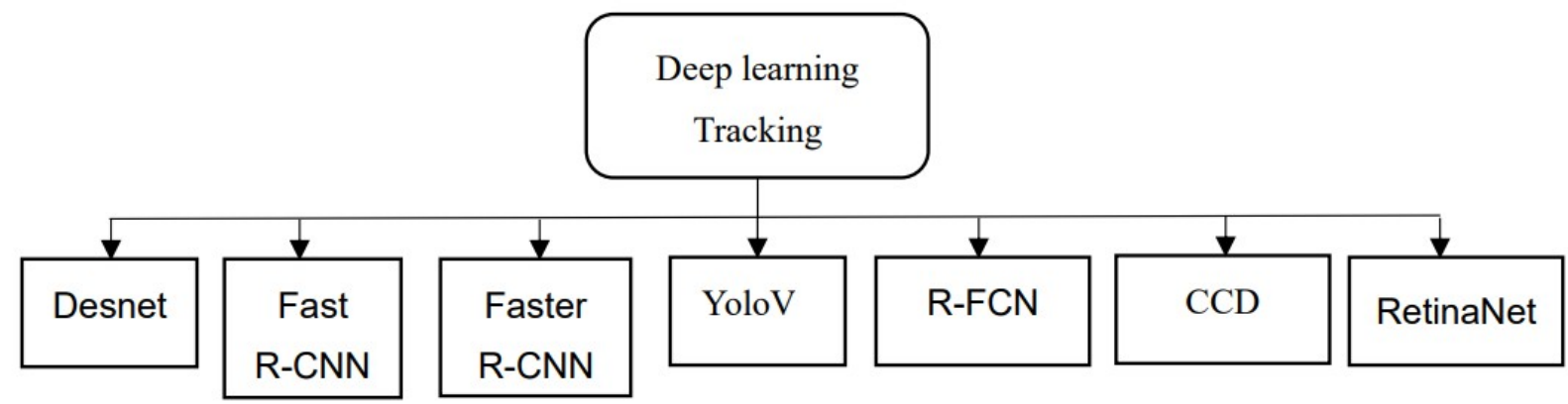

Рис. 4. Нейронные сети, которые можно использовать в процессе отслеживания сорняков

Сложность построения нейронных сетей заключается в том, что при выборе модели многие параметры зависят от задачи, количество входных данных, наличие времени и вычислительной мощности, что делает каждую работу уникальной, ввиду этого невозможно правильно смоделировать сеть изначально. Необходимо использовать, как можно больше механизмов в предварительной обработке. Общим недостатком всех исследовательских работ является отсутствие полноценной многолетней верификации результатов исследований. При распознавании сорняков на поле можно выделить два основных момента. Первое - это сложность, которая продиктована погодными условиями, наличие всевозможных вариантов различные физические места расположения сорняков. Второй - скорость распознавания и количество возможных объекты для обнаружения. В исследовании, описанном ранее, не было ссылок на веб-сайт Kaggle, это лучшее место для анализа производительности разработанных моделей широкой сети. Соревнования помогут дать реальную картину оценки моделей машинного обучения для каждого случая. Кроме того, в исследованиях нет ссылок на image.net. При этом скорость распознавания предметов в произведениях не раскрывается. Чтобы увеличить скорость распознавания и увеличить количество отслеживаемых объектов, помимо увеличения мощность компьютера, необходимо применять нестандартные действия, например, использование двух графических 
процессоров в стерео или использование нескольких графических процессоров в алгоритме обнаружения и комбинирование различных моделей нейросетей.

\section{References}

1. Ip, P., \& Ang, L. (2018). Big data and machine learning for crop protection. Computers and Electronics in Agriculture, 151, 376-383

2. Wang, A., Ang, W., \& Seng, K. (2019). A review on weed detection using ground-based machine vision and image processing techniques. Computers and Electronics in Agriculture, 158, 226-240

3. Nejati, H., Azimifar, Z., \& Destain, M. (2011). Weed detection in 3D images. Precision Agriculture 12(5), 607-622

4. Nejati, H., Azimifar, Z., \& Zamani, M. (2008). Using Fast Fourier Transform for Weed Detection in Corn Fields. IEEE International Conference on Systems, Man and Cybernetics, 1215-1219

5. Rueda-Ayala, V., Gerhards, R., \& Rasmussen, J. (2010). Mechanical weed control. Precision Crop

Protection - the Challenge and Use of Heterogeneity, 45, 279-294

6. Utstumo, T. (2018). Robotic in-row weed control in vegetables. Computers and Electronics in Agriculture, 154, 36-45

7. Alba, O., Syrov, L., \& Shirtliffe, S. (2019). Increased seeding rate and multiple methods of mechanical weed control reduce weed biomass in a poorly competitive organic crop. Field Crops Research,6, 245 8. Melander, B., Lattanzi, B., \& Pannacci, E. (2015). Intelligent versus non-intelligent mechanical intrarow weed control in transplanted onion and cabbage. Crop Protection, 72, 1-8

9. Syrov, L. (2016). Effects of mechanical weed control or cover crop on the growth and economic viability of two short-rotation willow cultivars. Biomass and Bioenergy, 91, 296-305

10. Panqueba, B., \& Medina, C. (2016). A computer vision application to detect unwanted weed in early stage crops, 4, 41-45

11. Reddy , R., Laasya,G. and Basha, M., 2017. Image Processing for Weed Detection, 5, Issue 4, 485489

12. Vikhram, G., Agarwal, R., \& Prasanth, V. (2018). Automatic Weed Detection and Smart Herbicide Sprayer Robot. International Journal of Engineering \& Technology, 7, 115-118

13. Pasha, I., Reddy, A., \& Mahima, P. (2018). DESIGN OF A WEED DETECTION SYSTEM FOR COTTON FIELD. International Journal of Pure and Applied Mathematics, 118, 1314-3395

14. García-Santillán, I., \& Guerrero, J. (2018). Curved and straight crop row detection by accumulation of green pixels from images in maize fields. Precision Agriculture, 19, Issue 1, 18-41

15. Murawwat, S. (2018). Weed Detection Using SVMs. Engineering, Technology \& Applied Science Research, 8, 2412-2416

16. Pulido-Rojas, C. (2016). Machine vision system for weed detection using image filtering in vegetables cropssa. Revista Facultad de Ingeniería, 56, 356-360

17. Bhongal, K., \& Gore,S. (2017). USING IMAGE PROCESSING TECHNIQUES AND SMART HERBICIDE SPRAYER ROBOT, 7, 10-16 
18. Michen, S., \& Lawer, A. (2019). Deep learning n agriculture, 187, 278-291

19. Ambika, N., \& Supriya, P. (2018). Detection of Vanilla Species by Employing Image Processing Approach. Procedia Computer Science, 143, 474-480

20. Ferreira, A., Freitas, D., Silva, G., Pistorib, H., \& Folhesc, M. (2017). Weed detection in soybean crops using ConvNets. Computers and Electronics in Agriculture, 143, 314-324

21. Sabanci, K., \& Aydin,C. (2016). Smart Robotic Weed Control System for Sugar Beet. J. Agr. Sci. Tech, 19, 73-83

22. Young, S. (2018). Future Directions for Automated Weed Management in Precision Agriculture. , Automation: The Future of Weed Control in Cropping Systems, 6, 249-259

23. Frasconi, C., \& Fontanell, M. (2014). Design and full realization of physical weed control (PWC) automated machine within the RHEA project. Proceedings International Conference of Agricultural Engineering, 134-143

24. Kulkarni, V. (2013). Advanced Agriculture Robotic Weed Control System. International Journal of Advanced Research in Electrical, Electronics and Instrumentation Engineering, 2, Issue 10, 5073-5081 25. Bakhshipour, A. (2017). Weed segmentation using texture features extracted from wavelet subimages. Biosystems Engineering, 157, 1-12

26. Kargar, A. (2013). AUTOMATIC WEED DETECTION AND SMART HERBICIDE SPRAY ROBOT FOR CORN FIELDS. Robotics and Mechatronics (ICRoM), First RSI/ISM International Conference, 451-470

27. Potena, C. (2016). Fast and Accurate Crop and Weed Identification with Summarized Train Sets for Precision Agriculture. International Conference on Intelligent Autonomous Systems IAS. Intelligent Autonomous Systems, 14, 105-121

28. Slaughter, D., \& Giles, D. (2018). Autonomous robotic weed control systems: A review. Computers and electronics in agriculture, 61, 63-78

29. Ospina, R. (2018). Smart Agricultural Vehicle by Integrating Motion Model with Machine Vision Data. Dissertation. Hokkaido University Collection of Scholarly and Academic Papers: HUSCAP 30. Dyrmann, M. (2015). Automatic Detection and Classification of Weed Seedlings under Natural Light Conditions. Thesis for: PhD. DOI: 10.13140/RG.2.2.29212.18560

31. Alba, O. (2019). Increased seeding rate and multiple methods of mechanical weed control reduce weed biomass in a poorly competitive organic crop. Field Crops Research, 245, 107-117

32. Bah, M. (2018). Deep Learning with unsupervised data labeling for weeds detection on UAV images. Remote Sensing 10(11), 15-17

33. Priya, S. (2019). Identification of Weeds using Hsv Color Spaces and Labelling with Machine Learning Algorithms. International Journal of Recent Technology and Engineering (IJRTE) ISSN: 22773878, 8, Issue-1, 1781-1786

34. Potena, C. (2019). Fast and Accurate Crop and Weed Identification with Summarized Train Sets for Precision Agriculture. Intelligent Autonomous Systems 14: Proceedings of the 14th International Conference IAS-14,105-121 
35. Ahmed, F. (2019). Machine Learning for Crop and Weed Classification. Performance Analysis of Support Vector Machine and Bayesian Classifier for Crop and Weed Classification from Digital Images. World Applied Sciences Journal, 12(4), 432-440

36. Sharma, P. (2019). Crops and weeds classification using Convolutional Neural Networks via optimization of transfer learning parameters. International Journal of Engineering and Advanced Technology (IJEAT) ISSN: 2249-8958, 8, Issue-5, 2285-2294

37. Zhang, W. (2018). Broad-leaf weed detection in pasture. IEEE 3rd International Conference on Image, Vision and Computing, 15-23

38. Huang, H. (2016). A fully convolutional network for weed mapping of unmanned aerial vehicle (UAV) imagery Huasheng. PLoS ONE, 450-470

39. Hameed, S. (2018). Detection of Weed and Wheat Using Image Processing. Conference: 2018 IEEE 5th International Conference on Engineering Technologies and Applied Sciences (ICETAS), 34-39 40. Wang, S., \& Liu, H. (2018). Low-Altitude Remote Sensing Based on Convolutional Neural Network for Weed Classification in Ecological Irrigation Area. IFAC-PapersOnLine, 51, Issue 17, 298-303 41. Huang, Y. (2019). UAV Low-Altitude Remote Sensing for Precision Weed Management. Weed Technology, 32, 1-5 